recommend that all the cattle in the herd be inoculated with not less than $20 \mathrm{cc}$. of glycerinated bile, $30 \mathrm{cc}$. of strong serum, or $200 \mathrm{cc}$. of defibrinated immune blood. The strength and endurance of the immunity conferred by these inoculating materials is proportionate to the amount injected; hence, if the disease is prevalent in the neighbourhood, larger doses may be used, or an injection of fresh pure bile may be given ten to fifteen days later. This will confer a lasting immunity on the cattle, and obviate the loss arising from the inoculation, or risk of introducing the disease.

\title{
PSEUDO-TUBERCULOSIS IN SHEEP ${ }^{\text {I }}$
}

\section{(LYMPHO-ADENITIS.)}

\section{By J. A. Gilruth, M.R.C.V.S., Chief Veterinarian and Bacteriologist, New Zealand.}

THE most frequent cause of condemnation of sheep, as reported by our inspectors at the various abattoirs and meat-export slaughterhouses, is an affection which consists primarily of localised imflammation followed by suppuration resulting in the formation of nodules, consisting of a fibrous capsule varying in thickness from one-eighth in. to one-quarter in., containing purulent material of varying consistency, and characterised by a distinctly greenish colour. Here it is as well to point out that these nodules are in no way associated with degenerated caseated and sometimes calcified hydatid cysts, which are readily distinguished by their greyish colour and the facility with which the degenerated centre can be enucleated, leaving the distinct cyst wall.

\section{NOMENCLATURE.}

Other observers who have studied the disease have preferred terms which appear to have been suggested solely by the affection of the lymphatic glands-hence the names "lymph-adenitis" (Norgaard and Mohler), "caseous adenitis" (Cherry and Bull), etc. As these terms tend to give a misleading conception of the nature of the disease, as well as of the situation of the characteristic lesions; and, what is more important, because of the close similarity both macroscopically and microscopically, as well as their usual location in the regions affected by tuberculosis, I prefer the term "pseudo-tuberculosis," which, while being open to objection, certainly gives some idea of the nature of the disease, the basis on which all terminology should be established.

\section{SITUATION.}

\section{Lymphatic Glands.}

The most common seat in the carcase proper is undoubtedly the lymphatic glands, particularly those of the precrural, prescapular, and popliteal regions; but the various other glands may be affected, either primarily or secondarily. In no case, however, so far as I can ascertain, have the pharyngeal, parotid, or sublingual lymph glands been

1 Reprinted from the Chief Veterinarian's Report for 1901-1902. 
found affected. Nodules possessing all the characteristics have been found in other portions of the carcase proper, such as the udder, the intermuscular tissue, \&c.; in one instance which came under my own observation an abscess the size of a bean being situated in the centre of a "chop."

\section{Viscera.}

For a considerable time I was of opinion that this was a disease practically confined to the lymphatic glands, but experiments have proved that the condition variously described in reports as "septic pneumonia," "septic pleurisy," "suppurative pleuro-pneumonia," \&c., is the same disease, due to exactly the same micro-organism. So far I have no evidence that the disease ever affects to any great extent other organs, such as the spleen, liver, kidneys, and intestines, though it may be that further observations will lead to a different conclusion.

\section{POST-MORTEM APPEARANCES.}

So far as we are aware, the disease when contracted naturally is never fatal, at most in long-standing cases a condition of cachexia resulting. The only evidence regarding the general distribution of the lesions we have is obtained from animals killed for human consumption, particularly at the meat export slaughter-houses.

The following interesting short note by $\mathrm{Mr} \mathrm{A}$. M. Paterson regarding the three types of pleurisy he has encountered in Southland is worthy of record :-

\section{"RE PLEURISY AND PNEUMONIA IN SHEEP.}

"In my work I have come across what appear to be three distinct types of pleurisy. Pneumonia pure and simple is very rare. One type of pleurisy is that which succeeds chills and exposure to wet. It is met most commonly in young sheep shortly after a spell of cold wet weather. About 2 per cent. of given mobs are thus affected after weather such as I have indicated. Adhesions are always present, and are usually extensive. Pneumonia is always present in these cases, but there is an entire absence of pus. Another type, which I have been in the habit of calling in my returns 'septic pleurisy,' is very frequently met with. This is common to all classes of sheep except the very oldest (I have not seen a single case at the... Canning Works, where they kill mostly old ewes). This condition seems to me to be of a specific nature. Adhesions are usually limited to the immediate neighbourhood of an abscess which is usually on one side or the other, seldom on both. The material in the abscess is to all appearances similar to that found in caseous nodules. Sometimes adhesions extend beyond the abscess, complicating the whole of the pleura on the affected side, but I have not seen more than one abscess in any case. The third type seems to be confined entirely to old sheep. There are no adhesions ; the parietal pleura are not involved; the condition seems to be extremely passive. The pulmonic pleuræ are thickened and closely studded over with small white fibrous nodules about the size of small peas. I have cut many of these open, but have never detected any pus or caseation. The whole 
lung appears darker than usual when first taken from the sheep, and it does not brighten much on exposure. When cut into the lungsubstance shows numerous fine blood streaks extending from the pleural surface an inch or so into the tissue of the lung, where they shade away into fine threads and disappear.

"... The carcase does not set well, and the cellular tissue in inguinal region is emphysematous at the end of twenty-four hours. Before slaughter nothing is noticed about an animal affected. It is usually well nourished, and does not appear dull, although an occasional cough is observed."

The first type mentioned may be, as suggested, simply due to chills, though, being noted in all parts of the colony, the probability is that the origin is specific. In fact, considering that the three conditions are to be found at three different periods of the animal life, it is most likely they are three stages of the same disease, the latter being an attempt at complete recovery. However, $\mathrm{Mr}$ Paterson is arranging to secure specimens for definite examinations, and I trust to be able to decide shortly.

The following report by $\mathrm{Mr}$ Kerrigan, M.R.C.V.S., Government Veterinarian at Ashburton, Canterbury, on the occurrence of purulent and caseous conditions in the pleura and lungs of sheep gives a careful and complete description of the pathological lesions as met with in a large number of cases in the ordinary work of meat inspection:-

\section{“ PLEURISY AND PNEUMONIA IN SHEEP.}

"In my monthly reports I have used the term 'septic pleurisy' to convey the idea that certain animals were condemned for an inflammation of the pleural surfaces, which had terminated in the formation of pus. This term was concise, and suited the limited space allowed in the report forms, and, although correct in a broad sense, still it did not distinguish the various causes which produced this septic condition of the pleura. The following are the various conditions found present :-

$$
\text { " ' Septic' Pleurisy. }
$$

“I. Pleurisy Proper, temninating in Suppuration-Lambs and old ewes are the subjects generally affected by the above. This condition is found frequently present on the right side, occasionally on the left, and rarely on both. It is generally situated somewhere on the pleura covering the middle thirds of from the fifth to the tenth rib, and the corresponding part of the visceral pleura. The extent of the affected part varies, as sometimes it could be covered by a penny, while in other cases the whole of the above-mentioned area is affected. On rare occasions two patches of septic pleurisy were found present on the same side. The area affected is well defined, being circumscribed by fibrinous adhesions, and these adhesions exist to a greater or less extent throughout the part. The purulent material is viscid, of dirtygrey colour, and sometimes sanguineous. When the pus is scraped off, the pleura is found to be thickened. There may be slight congestion of the lungs under the affected part; but otherwise the lungs appear healthy.

"2. Septic Pleurisy associated with Nodules in the Lungs, and also 
with Nodules in the Supra-sternal Glands.-These nodules are similar in appearance to those I have already described under 'Caseous Nodules'-as found situated in the regions of lymphatic glands. Merinos, half-breds, and old ewes of various kinds are the subjects affected by this condition. The nodule apparently gives rise to an inflammation of the pleura, which terminates in suppuration. The pus has generally the same greenish appearance as the contents of the nodule. In the case of a nodule in the lung accompanying the pleurisy, it is generally situated immediately under the pleura.

" 3 . In connection with septic pleurisy I must also mention a condition, sometimes present in lambs, in which a 'false membrane' is found adhering between the visceral and costal pleuræ. In most cases both sides are equally affected. The part generally affected is the lower two-thirds of the pleura lining from the first to the tenth rib, and the corresponding visceral pleura. The membrane can be stripped off-sometimes with difficulty-and at some parts it may be about a quarter of an inch thick. To the naked eye it has all the appearances of a network of tissue infiltrated with pus; however, it is only on rare occasions that pus is really present. The membrane is very elastic, and when pressed between the fingers the contents of the network do not stain the fingers like pus, but rather give them an oily feeling. The surface of the pleura looks rough where the membrane is attached to it; but at some parts under the membrane no attachment exists, and in those places the pleura may look healthy. The appearances of this condition are so deceptive to the unaided eye that it is only by handling the affected parts and membrane that it can be decided whether the appearances are due to an infiltration of purulent material or probably a fatty degeneration of the false membrane.

"The following lists give the numbers of sheep and lambs condemned at Fairfield for septic pleurisy during the period included from the I Ith May I901 to the 8th March I902:-

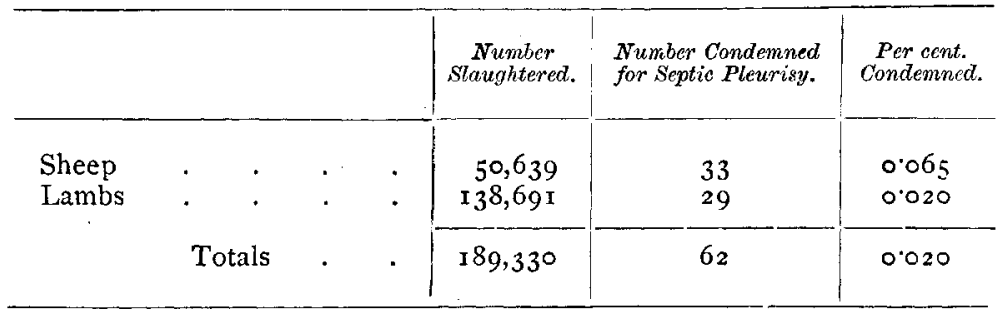

"In about 60 per cent. of the above condemned animals the septic pleurisy was accompanied by nodules in the lungs and supra-sternal glands.

"The total number of sheep and lambs condemned out of the above numbers for all diseases was 244 .

$$
\text { “' Septic' Pneumvnia. }
$$

"The following are the septic conditions I have found present in the lungs of sheep:-

"I. A Septic Condition of the Lungs due to Large Nodules containing. 
Viscid Greenish-looking Pus or Half-caseated Material.-There may be from one to six nodules present. They vary in size from a bean to a walnut, are ovoid in form, and surrounded by a capsule. They resemble in every way the nodules already described under 'Caseous Nodules.' Sometimes this condition of the lungs is accompanied by a nodule in the prescapular or precrural regions; but this is by no means common. They are generally situated near the pleura of the costal surface of the lung and just opposite the root ; but they are sometimes present at the base of this organ, and occasionally at the apex. As already mentioned, they frequently cause a septic condition of the pleura. These nodules are found present in old ewes, chiefly Merinos and half-breds.

"2. A Septic Condition of the Lungs due to Small Nodules about the Size of No. 2. Shot.-For some time the appearances of these nodules gave me the impression that they were caused by some parasite; but the same greenish-looking pus or caseous material as described above and under 'Caseous Nodules' is found present in a number of them, hence this condition may be due to the same cause as the large caseous nodules. The different appearance, or rather size, of the small and large nodules and the manner in which they affect the lung may be due to the cause (or organism) of the disease finding its way to this organ by different channels. This condition is found present in sheep and in lambs. In many ways these small nodules resemble secondary pulmonary tuberculosis propagated through the medium of the blood-vessels. They are sharply defined from the surrounding pulmonary parenchyma, and generally remain distinct, as it is only occasionally that two nodules seem to coalesce. They appear just under the visceral pleura, but are also distributed almost equally throughout the tissues of the lung. The following different stages may be seen in the same lung, just under the pleura :-

"(I.) A small congested area of a reddish-brown colour, sharply defined and hardly perceptible under the finger. The condition gradually changes into the next stage.

"(2.) A sharply defined nodule similar in size to the congested area in stage No. I. It is easily perceptible under the finger, and has the colour of starch as prepared for use on linen. When a nodule in this stage is cut it is sometimes almost like cartilage under the knife. Occasionally one is found in a calcareous state.

"(3.) In the centre of the above cartilaginous-like nodule a small yellow point appears, easily visible to the naked eye. This gradually increases in size.

"(4.) In this stage the nodule is made up of a capsule filled with greenish-yellow-sometimes yellow-caseous-looking material.

"Although the nodules vary a little in size, still they seem to remain about the same size throughout the above four stages. More nodules are found in the second and third stages described above than in the first and fourth. They are not associated with any pleural adhesions. The number of nodules in a lung varies, as in some cases there are only a few, while in others they are very numerous. In the right lung of an old ewe (Lincoln and Leicester cross) 275 nodules were present just under the pleura- 134 on the costal surface, 84 on the internal or mediastinal surface, and 57 on the base-and they were also distributed about equally throughout the lung-tissue. I 
have sometimes seen a lung with four to five times the above number of nodules present.

"Occasionally cirrhotic-looking patches of lung-tissue are present, about $\frac{1}{2}$ to I cubic inch in size. They are generally depressed slightly below the level of the surrounding tissue, and are pale in colour when compared with the rest of the lung. When these patches are cut they have an indurated feeling under the knife, and in them the nodules are more numerous than usual. The change in the lung in this case is probably due to an increase of interstitial tissue on account of the irritation caused by the increased number of these nodules.

"Situations of the nodules: Immediately under the pleura of lung and throughout the lung-tissue; but they generally appear first on the costal surface of the lung, close to where the diaphragmatic border joins the vertebral border.

"From the examination I have made of a large number of lungs, I believe that about 40 per cent. of the sheep passed through Fairfield are affected with this condition, and I would not be surprised to find the percentage much more. Some lots of sheep and lambs may be almost free from these nodules, while in others it is difficult to get one lung without them.

"The following lists of three small lots give some idea of the number affected with small nodules immediately under the pleura :-

\begin{tabular}{|c|c|c|c|c|}
\hline & $\begin{array}{c}\text { No } \\
\text { Nodules. }\end{array}$ & $\begin{array}{l}\text { Under } \\
\text { Twenty } \\
\text { Nodules. }\end{array}$ & $\begin{array}{c}\text { Over } \\
\text { Twenty } \\
\text { Nodules. }\end{array}$ & Total. \\
\hline $\begin{array}{l}\text { Late lambs, half-bred English } \\
\text { Leicesters } \\
\text { Four-tooth wethers, mixed breeds } \\
\text { Ewes, mixed breeds . }\end{array}$ & $\begin{array}{r}127 \\
28 \\
-\end{array}$ & $\begin{array}{r}39 \\
9 \\
4\end{array}$ & $\begin{array}{r}7 \\
37 \\
21\end{array}$ & $\begin{array}{r}173 \\
74 \\
25\end{array}$ \\
\hline Totals . & 155 & $5^{2}$ & 65 & 272 \\
\hline
\end{tabular}

"These nodules are frequently met with in the mediastinal glands, especially in Merinos and half-breds. I have occasionally seen them in the sublumbar glands, and on rare occasions I have condemned a carcase with numbers of them along the back, and at other times along the abdomen just under the skin.

"3. A Septic Condition of the Lungs due to Abscess-like Cavities containing Yellowish-looking Fus.-These are found present in old ewes, and have not the appearances of No. I and No. 2. They look like infarctions, and are generally accompanied with a septic condition of the mammary gland.

"4. Pneumonia Proper, terminating in Suppuration.-I have only met with a few doubtful cases of this kind in lambs, and also in sheep."

The following report by $\mathrm{Mr}$ Edgar is also interesting. It will be noted that he treats both the pulmonary and lymphatic lesions as the same disease :- 
"CASEOUS NODULES IN SHEEP.

"The most frequent cause of the condemnation of the carcases of sheep is the presence of suppurating foci in the internal organs and the carcase. These lesions are most commonly found in the lung and pleura, but may also be present in other parts of the viscera, and in the lymphatic glands and muscles of the body, the most common site being the lymphatic glands of the groin, the muscles of the thigh, and the substance of the mammary gland. . . .

"The lesion takes the form of an abscess or caseous tumour enclosed in a fibrous capsule, the contents of which consist of pus, creamy in consistency and of a pale-green colour. Later, by pressure and absorption of its liquid parts the pus becomes caseated, and the parts may heal. The lungs may contain one or more of these abscesses or nodules, and it has been my experience to find them always isolated, the intervening portions of the lung being in most cases healthy.

"When situated in the costal pleura they are less likely to become caseous or to heal, and there is more pus. In recent cases the pleura is brightly inflamed: in older ones it is thickened and attached to the lung by fibrous adhesions. Sometimes the abscess involves the costal muscles, and appears as a small bulging tumour between two ribs. The presence of these abscesses is usually confined to one portion of the body-most commonly the lungs and pleura-but it is by no means rare to find the muscles of the thigh or the lymphatic glands involved at the same time. . . .

"That the disease causes the sheep little inconvenience or detriment of bodily health is shown by the carcase being usually fat ; in fact, all the cases that have come under my notice have been in sheep slaughtered for freezing or for local consumption. ... That this disease is fairly general throughout the North Island is shown from the fact that a certain number of sheep from all parts are condemned as being unfit for human food. ... I have noted no symptoms. Sheep always appear healthy before slaughter. I have found the average number affected to be one in one thousand sheep. It has been my experience to find the disease more prevalent among aged ewes and broken-mouthed sheep than in any other class. I think this may show that the wound-infection takes place while grazing when the teeth are absent or much worn. Lambs are the least affected. This may be due to the fact that their mouths are unbroken."

\section{DISTRIBUTION.}

All parts of the colony seem to be more or less affected. While the disease may be found in any breed, it seems undoubted that the Merino is the more prone to be affected. In a long and interesting report, Mr Kerrigan, who has had much experience, states :-

"... These caseous nodules are usually found in the situation of lymphatic glands. In my experience I find that the nearer the sheep is bred to the Merino the greater is the percentage of animals affected with them ; however, they are found present in all classes of sheep and occasionally in lambs. I have seen a thousand sheep from a certain piace go through without the sign of a nodule; while, again, 
another flock of wethers from the same place and owner, but bred more to the Merino, have been found affected to the extent of $3 \frac{1}{2}$ per cent. . .. The percentage of animals affected varies very much, but I might particularly mention that the old Merino ewes have to be carefully inspected for them. The following numbers give an idea of the percentage condemned for these cysts. They include the lambs and sheep slaughtered at Fairfield from the time I commenced inspection at these works in May I9OI, to the first week in March I902 :-

\begin{tabular}{|c|c|c|}
\hline Numbers slaughtered during above & Lambs & Sheep. \\
\hline period $\quad \ldots$ & I $38,69 \mathrm{I}$ & 50,639 \\
\hline Numbers condemned for nodules. & 29 & 89 \\
\hline Percentages $\ldots \quad \ldots \quad \ldots$ & 0.020 & 0.175 \\
\hline
\end{tabular}

The total number of sheep and lambs condemned out of the above numbers for all causes was 244."

Other veterinarians who have experience in inspections, and have had opportunity of comparing its prevalence in different breeds, agree that the disease is much more prevalent in the Merino than in others.

\section{PATHOLOGY.}

The lesion commences by the arrest of the specific bacillus, generally in a lymph-gland, where one or more are surrounded by and included within the phagocytes. The micro-organisms multiply within the cells, and ultimately cause the degeneration and death of the latter. Simultaneously a slow chronic inflammation occurs around the focus of attack; there is proliferation of connective tissue-cells and the formation of more or less new fibrous tissue. As the process spreads outwards the centre degenerates, and the protecting wall increases in thickness. In fact, all the phenomena of the pathology of true tuberculosis in a gland occurs, with the exception of the formation of giant cells. The degenerated centre of the nodule assumes a greenish tint, especially distinct at the time of exposure by the knife, but gradually becoming greyer afterwards. In older tumours in the centre of the purulent or caseous mass (for the consistency varies from that of cream to that of cheese in different tumours) there are usually present no bacilli which can be demonstrated by the microscope or by cultural methods.

The pulmonary lesions I have not had much opportunity of observing in the earlier stages. As to whether the primary lesion is ever in the lung or the pleura, definite information is wanting; but it will be observed that my feeding-experiment in the sheep had a negative result, while Norgaard and Mohler were equally unsuccessful with their feeding-experiments. In addition, the question as to whether the pleurisy is secondary always (most of my evidence tending to this conclusion), or whether it may occasionally be primary to the lung-affection, remains to be settled. Generally, however, the first nodules appear near the pleura, most frequently in the superoexternal region. I have personally observed them as small as a millet-seed, readily overlooked and possessing a slightly greenish tint, even through the intervening pleura. Gradually the pleura becomes involved, a fibrinous exudate results, and later on, provided 
practical recovery with a slight permanent adhesion does not result, a quantity of pus or caseous material develops in the pleural cavity, localised always more or less by a thick fibrous wall.

As evidencing the extent to which the disease may attain, and without apparently injuring the health of the animal, under ordinary circumstances, the following note of a case by $\mathrm{Mr} \mathrm{H}$. S. Reid is interesting:-

"The subject, a wether in good condition, showed no apparent symptoms during life. On opening the thorax the left lung was found to be intimately adherent to the thoracic wall. Two-thirds of the lung-tissue were simply converted into a large abscess filled with inspissated pus, the remaining portion of the lung being hepatised, and showing the usual pneumonic lesions. The right lung was normal, except in the posterior portion of the lower border, which was slightly affected with pneumonia."

\section{THE MICRO-ORGANISM.}

The organism with which I have conducted my investigations was isolated from pus secured from a precrural lymphatic abscess forwarded in sealed Pasteur pipettes by $\mathrm{Mr}$ D. H. Rait, Government Veterinarian, then engaged in the inspection of meat at the Belfast freezing-works. These specimens had been so carefully secured that I was able with ease to prepare pure cultures therefrom.

The specific organism is a short irregular-shaped bacillus, varying in size and appearance from a coccus to a very short bacillus-i.e, from 0.5 to $I$ micron in length and about $0_{3} 3$ in breadth. In the pus of an early abscess the bacilli are frequently within more or less degenerated leucocytes, especially those in scrapings from near the periphery of the abscess or the lining of the fibrous capsule. In the centre of an old abscess the bacilli are usually very degenerated and irregular, occasionally being quite absent. Frequently the organisms are found in pus in chains which may almost be mistaken for streptococci, though careful examination demonstrates that these chains are composed of short cocco-bacilli extremely small even in their longest diameter, and arranged side by side, not end to end. The bacilli are readily stained by the method of Gram. When pus is stained by a simple stain, such as methylene blue, thionin, or weak fuchsin, they are with difficulty observed consequent upon their small size. The best method of demonstration is to stain pus by Gram's method and counterstain with eosin or weak fuchsin.

\section{Growth in Media.}

Serum Cultures.-The bacilli grow most readily and exhibit the most distinctive characteristics on solidified clear ox-blood serum. Separate colonies appear after twenty-four hours at $37^{\circ} \mathrm{C}$. as small greyish points. These gradually increase in size for the next two days, when they reach the diameter of $3 \mathrm{~mm}$., assume a distinctly yellowish tint, and have a raised darker centre and irregular edge. The distinctive characteristic, however, is not observed on examining the colonies on the free surface of the serum-slope. If examined through the clear solidified serum a yellow feathery zone is found to radiate from the edge of the colony into the substance of the serum and on each side 
of the surface-growth. This zone or aureole is very peculiar and striking. Naturally it is only to be observed in carefully prepared serum which is sufficiently clear to be transparent. Streaks on slope serum show a thin, slightly yellowish growth with irregular borders after twenty-four hours at $37^{\circ} \mathrm{C}$. It is rarely till the second or even the third day that the characteristic zone makes its appearance. The breadth of the streak culture gradually increases, but, as a rule, never exceeds $4 \mathrm{~mm}$. The aureole, however, increases till practically the whole of the serum is occupied by the growth. In the condensation liquid growth takes place readily, the liquid becoming rapidly cloudy with a dirty yellowish-white appearance and a considerable dense yellow deposit at the bottom. In subcultures on serum incubated at $37^{\circ} \mathrm{C}$. a faint growth can be observed six hours after inoculation. In liquid serum the bacillus grows very readily. A flocculent yellow scum forms on the surface, and in two days reaches a thickness of $2 \mathrm{~mm}$. The rest of the serum remains cloudy and of a whitishyellow-tinge, with a large yellow deposit at the bottom of the liquid. If the tube be shaken the yellow scum can be easily mixed with the fluid, but much gradually returns to the surface. The bacilli, when grown on serum, assume the form of cocco-bacilli, generally in pairs, slightly shorter and narrower than those found near the wall of an abscess, but may vary from almost a coccus to a bacillus nearly as long as that of typhoid.

In sheep-serum the growth is entirely distinct from that of ox-serum, there being no pigmentation. The growth is greyish-white, and the definite feathery zone of the ox-serum is absent or barely observable. In liquid serum growth is very rapid, and similar to that already described in liquid ox-serum, with the exception that the colour is a greyish white.

Broth Cultures-If carefully inoculated, after twenty-four hours a thin scum may be observed on the surface of the liquid, with a powdery deposit on the sides and at the bottom of the tube. This scum is generally irregular on the surface, and readily broken down into a flocculent mass, little of which returns to the surface on settling. If another tube be inoculated with a portion of the scum it rapidly grows, and no turbidity of the broth is produced. If broth be inoculated in the depth, little or no scum is produced. A very faint turbidity is apparent twenty-four hours after inoculation, but this soon clears, leaving only a deposit at the bottom of the tube and a faint powdery mass adherent to that side which is lowest on the plane of the slope, while the liquid remains clear. A broth culture examined under the microscope shows the bacilli in masses or rows, which are apparently difficult to break. When in rows the bacilli look not unlike very smail streptococci, but careful examination shows each organism to have a long and narrow diameter, the bacilli lying side by side, as it were, and not end to end.

Agar Cultures.-On ordinary agar I found it difficult at first to secure any growth when inoculated in the ordinary way with pus by means of a needle. After subcultures were made on serum, however, a good growth could be obtained on slope agar. The growth consists of, after twenty-four hours, a thin powdery-looking streak, with irregular borders. On agar subs, again, the growth increases slightly in breadth and thickness, its borders showing the irregular crenated 
appearance characteristic of cultures of this organism. The colonies appear as greyish-white points with raised centre, and do not attain the maximum size for ten or twelve days, when they are from $4 \mathrm{~mm}$. to $6 \mathrm{~mm}$. in diameter, with crenated borders. In streak-agar sub-cultures the growth after the lapse of a week consists at $37^{\circ} \mathrm{C}$., when viewed through the slope agar, of a narrow central grey-brown line, on each side of which is a zone of a thick white growth, external to which is a zone of semi-transparent growth with distinctly crenated borders.

In all cultures the growth is very tenacious and cohesive, under the microscope the bacilli generally appearing in masses, or chains. The bacillus, when grown on agar or glycerine agar, is very short, almost resembling a streptococcus both in size and arrangement, and at times is almost indistinguishable from cocci with an ordinary one-twelfth oil-immersion lens. When very carefully examined, however, it is seen to be somewhat longer than appears at first sight.

Glycerine agar as a medium is not favourable, the growth being not so characteristic or so distlnct as on ordinary agar.

In gelatine at room-temperature a growth, very fine and with no characteristic appearances, is visible in about five to eight days. It grows equally well in slope or stab culture.

On potato, either ordinary or with glycerine, I have not succeeded in securing any growth.

In milk the bacillus grows readily at $37^{\circ} \mathrm{C}$., forming a thick, brownish deposit at the bottom of the tube. The milk is not altered in reaction, as shown by cultures made in litmus milk. The bacilli are found in groups, and are very short, being in certain cases almost indistinguishable from cocci.

\section{EXPERIMENTS.}

Sheep I.-Inoculated in thigh with $0^{\circ} \mathrm{I}$ cc. pus from a precrural lymph-gland forwarded by Mr Rait.

Practically no evidence of discomfort beyond a very slight lameness for the first two days was noticed in this animal. The animal remained in good condition, and was killed four months later.

Post-mortem Examination. - At original seat of inoculation a small mass of greenish caseous matter was present surrounded by a fibrous capsule about $\frac{1}{4}$ in. thick. The popliteal, precrural, and pelvic glands of the same side were enlarged and caseous, with the usual capsule surrounding the central caseous greenish mass of degenerated material. Other lymphatic glands of the body normal. Lungs : Under the pleura of both lungs were a number of nodules varying from the size of a pea to that of a marble, of a greenish-translucent tint when fresh, and surrounded by a purple zone. On collapse of the lung these nodules became more prominent. Throughout the lung-tissue generally a number of similar nodules were present, but the greatest number were situated just underneath the pleura. Each nodule consisted of a greenish caseous centre surrounded by a thick fibrous wall, which gradually merged into healthy lungtissue. There was no pleurisy, and the thoracic lymphatic glands were healthy. From the glands and Iung the characteristic organism was isolated. 
Sheep 2.-Inoculated in flank with $0.5 \mathrm{cc}$. three-days-old culture in serum condensation liquid.

Next day the animal was carrying the inoculated leg, and showing slight swelling and inflammation at seat of inoculation. The following morning she' was found lying on her side dead, with blood-tinged froth issuing from the nostrils.

Post-mortem Examination.-Animal in good condition; small quantity of subcutaneous odema at seat of inoculation. The spleen was enlarged and pulpy, the contents being of a tarry consistency. The alimentary tract was filled with ingesta. The abomasum exhibited on its mucous surface small patches of inflammation, each with its centre slightly ulcerated. The pleural cavity contained about a quart of sero-sanguineous effusion. The kidneys were slightly congested. Other organs normal.

Microscopical Examination of smears disclosed no organisms in any of the tissues, beyond a large number present in the subcutaneous cdema at the seat of inoculation, and these chiefly within leucocytes. Pipettes containing spleen-pulp, pleural effusion, and blood were placed at $37^{\circ} \mathrm{C}$., but did not develop the characteristic bacillus, while serum-tubes inoculated with the same material remained sterile. Tubes inoculated with the subcutaneous effusion developed the characteristic growth of the bacillus in a state of purity.

Guinea-pigs I and 2.-Each inoculated with $\mathrm{O} I \mathrm{cc}$. of the same culture as that used for sheep 2. As during the process of inoculating No. I the saphena vein was accidentlly punctured with the needle, No. 2 was inoculated as a control.

In twenty-four hours both animals were dull, had lost appetite, and showed slight swellings at the seat of inoculation. By forty hours both were very sick, breathing rapidly, temperature raised, and huddled together in a corner of the hutch. Forty-eight hours after inoculation guinea-pig 2 died.

Post-mortem Examination.-2: Small quantity of subcutaneous oedema around seat of inoculation, with congestion of the area. Intestines and mesenteric glands congested. Stomach abnormally distended, being filled with ingesta. Towards pyloric extremity on the greater curvature was an area of inflammation extending from the mucosa to the peritoneum, the submucosa being at the centre thickened with gelatinous cedema. This inflammatory area was about $\frac{1}{2}$ in. in diameter, and generally merged into the healthy tissue. Spleen slightly enlarged. The peritoneal cavity contained a quantity of sero-sanguineous effusion. Other organs normal.

Microscopical Examination demonstrated large numbers of bacilli present in the cedema at the seat of inoculation, many being within leucocytes. Media inoculated from spleen, blood, and abdominal serosity remained sterile.

Guinea-pig I was found dead with rigor mortis well advanced fiftyfour hours after inoculation.

Post-mortem Examination revealed a condition similar to that of 2, with the exception that at the seat of inoculation there was present a small nodule of yellowish pus.

Microscopical Examination demonstrated a similar condition to that found in 2. Further investigation demonstrated that the organism was not present in other portions of the body. 
Guinea-pig 3.-Inoculated with $0^{\circ} \mathrm{O} 2 \mathrm{cc}$. fluid from bottom of serum culture, being a twenty-four-hours-old subculture from original culture.

In eighteen hours a considerable swelling with slight inflammation had developed at the seat of inoculation. At forty hours after inoculation the animal was seen lying on its side listless, and not moving when disturbed; breathing spasmodic; temperature subnormal. Death occurred forty-four hours after inoculation.

Post-mortem Examination showed a small area of pus about the size of a split pea near the seat of inoculation, with considerable cedema of the subcutaneous region of the thigh and the posterior portion of the abdomen. The stomach showed a similar lesion to that of the previous guinea-pigs. The spleen was normal; liver and kidneys slightly congested. No pleural or peritoneal effusion. Other organs normal.

Microscopical Examination.-Smears from pus and odema of leg showed numbers of the characteristic bacilli, chiefly within leucocytes, where they were very polymorphic, but all staining readily. Culture experiments resulted as in previous cases.

Sheep 3.- Inoculated on inner surface of fore leg above the carpus with $0.05 \mathrm{cc}$. three-days-old subculture on liquid serum from guineapig I.

Next day the animal walked very lame on the inoculated limb, and there was a slight swelling and inflammation at the seat of inoculation. Twenty-four hours after inoculation the leg was being carried, but the animal would run well on three legs. The temperature was normal. On the second day the swelling had increased and was extending up the leg. The temperature gradually rose till at fortyeight hours after inoculation it stood at $104.3^{\circ}$ Thereafter the sheep gradually regained its normal condition, a slight lameness of the inoculated limb being only observable, and this continued up to the time when slaughtered-three months later-for post-mortem examination. The swelling at and above the seat of inoculation still persisted, though the inflammation disappeared after the'first week. A few weeks before slaughter a small abscess burst above the seat of inoculation, and this was followed by other similar abscesses bursting on the inner aspect of the leg.

Post-mortem Examination.-Animal in good condition-in fact, almost fit to kill for mutton. A number of subcutaneous abscesses near the seat of inoculation extended around the leg above the knee. On the fascia of the muscles at the seat of the inoculation was situated a tubercular-looking mass of small caseous nodules, consisting of a dense fibrous capsule about one quarter in. in thickness, with a degenerated centre, greenish in colour, caseous in consistency, the majority of these nodules being about the size of a pea. In the subcutaneous abscesses the greenish material was more fluid than in the above tubercular-looking mass. The lymphatic glands near the centre of the radius were enlarged, the first of the group being of the size of a walnut, the remainder smaller. They were completely altered, no healthy gland-tissue being present, and consisted of a central greenish caseous mass surrounded by dense fibrous tissue. The brachial lymphatic glands were enlarged to the size of a walnut, and in a similar condition to those described above. 'The prepectoral glands 
were enlarged, but otherwise normal; while the subscapular group lying beneath the centre of the scapula were enlarged and caseated. Thorax: Fibrinous pleurisy was present in the infero-anterior half of the right side of the cavity (the opposite to the inoculated side), the pulmonary pleura being joined to the costal by strong adhesions. There was no pus present. Lungs: Under the pleura of both lungs, especially the supero-external half, were present a number of circular translucent pale patches varying from the size of a pea to that of a marble, surrounded by a narrow bluish zone. On pressure these nodules appeared circumscribed and resistant. On section they were found to consist of a central mass of the typical greenish-coloured thick cascous material, each surrounded by a dense fibrous capsule from one-eighth in. to one quarter in. in thickness. These nodules were not present in the deeper portions of the lung.

Other organs, including the thoracic lymph glands, were normal.

Microscopical Examination.-In all the abscesses the typical bacilli were found present in large numbers, especially near the wall. In the pulmonary abscesses, which were evidently those latest developed and were growing actively, many bacilli were found within leucocytes. In the pleuritic adhesions it was only with difficulty that a few bacilli could be demonstrated, and these were degenerated and chiefly within leucocytes. The organism was recovered by means of cultures from the glands of the leg and the lung nodules. The blood remained sterile, even after incubation in Pasteur pipettes, for forty-eight hours.

Sheep 4.-Fed after twenty-four hours' fast with $4 \mathrm{cc}$. five-days-old liquid serum culture made from original culture.

Animal showed no evidence of illness, and was killed four months later.

Post-mortem Examination.-Internally all organs normal, with the exception that the liver was badly affected with hydatids, the lungs being also slightly affected. The left prepectoral gland was enlarged to the size of a marble, with a pea-sized centre of typical greenish pus surrounded by a strong fibrous capsule a quarter of an inch in thickness.

[NotE.-It is improbable that this gland should have been alone affected by the ingestion of such a quantity of culture. It seems more reasonable to assume that this sheep became accidentally inoculated by pus from an abscess which had burst in one of the other experimental sheep running in the same enclosure, more especially considering the early stage of the disease.]

Guinea-pig 4.-In order to ascertain if the suspicion were correct that a toxin developed in the culture before inoculation was responsible for the death of Sheep 2 and Guinea-pigs I, 2, and 3, and not a toxin produced after inoculation, a liquid serum culture incubated at $37^{\circ}$ for seven days was filtered through a Pasteur-Chamberland filter, and of this $0.5 \mathrm{cc}$. was injected subcutaneously.

Beyond a slight swelling at the seat of inoculation for the first day, and profuse sweating of the whole of the inoculated limb and inferior surface of the abdomen (during which the temperature remained normal), no apparent pathological results ensued.

Sheep 5.- Inoculated in thigh with $0.25 \mathrm{cc}$. four-days-old broth subculture, as used for Guinea-pig 5 (see below).

The usual symptoms of lameness were evinced a few days after 
inoculation, and persisted slightly up till ten weeks later, when the animal was killed for examination.

Post-mortem Examination.-Near the seat of inoculation a small quantity of pus was present, with much dense fibrous tissue. The popliteal glands were much enlarged, and consisted simply of a thick fibrous envelope with a firm greenish caseous centre. The pelvic lymphatics of the same side each contained a pea-sized nodule of greenish pus, with a thick fibrous wall surrounded by apparently normal gland tissue. The other organs were normal.

Guinea-pig 5.-Inoculated with $O^{\circ} \mathrm{I}$ cc. four-days-old broth subculture.

In twenty-four hours there was a slight inflammatory swelling at the seat of inoculation, and twenty hours later the animal was found dead, rigor mortis being well advanced.

Post-mortem Examination.-Small nodule of pus at seat of inoculation, with surrounding subcutaneous cedema and congestion. Large intestines inflamed. Stomach congested at pyloric half similar to previous cases, though less marked. Other organs normal. Microscopical examination gave same results as in previous cases. Further bacteriological examination proved the blood, \&c., to be sterile, the organism being confined to the pus and codema at the seat of inoculation.

Goat (Aged Male).--One that had been caught from a wild herd and sent to the laboratory for examination of lice, with which he was infested.

Inoculated in flank with $0.25 \mathrm{cc}$. broth culture the same culture being used for Sheep 5 and Guinea-pig 4).

In twenty-four hours the animal was very sick, had lost all appetite, the back was arched, and the leg carried when moving. The temperature was $105^{\circ}$. He continued for two days in this condition, the temperature remaining above normal, after which gradual recovery took place, although slight stiffness of the inoculated limb remained, due to a large abscess which gradually developed above the seat of inoculation. Two months after inoculation the goat was killed for examination.

Post-mortem Examination.-Animal in good condition. At seat of inoculation, and extending over a radius of about 2 in., were situated a number of discrete, pea-shaped nodules, of firm consistency, consisting of a central mass of thick greenish caseous material surrounded by a dense fibrous wall. The first of the group of precrural glands was enlarged to the size of an orange, forming the tumour, the outline of which was visible before death, and which consisted of the typical greenish caseous material, in this instance much thinner in consistency and semi-fluid, almost pus-like, surrounded by a dense fibrous wall about a quarter of an inch in thickness. The remaining glands of the group were also enlarged and caseous, but not nearly to the same extent. Surrounding the largest tumour and attached to the outer surface were smaller nodules, varying from the size of a pin to that of a hazel-nut, and in the surrounding muscular tissues were a number of similar nodules, but with thinner and less dense walls. The pelvic glands of the same side were enlarged and caseous. It should be observed that many of the nodules of the leg, particularly the larger ones, when cut across. were found to consist of several 
caseous circular areas separated by dense fibrous tissue, and but for the greenish colouring of the degenerated centre exactly similar to lesions of tuberculosis. The spleen exhibited at the infero-anterior angle a patch of blood extravasation of about $I$ in. in diameter. Other organs and glands normal.

Microscopical examination of pus from the large abscess showed the presence of the characteristic bacilli, varying from almost a coccus to a fairly long bacillus. The bacilli in the smallest nodules were chiefly very short cocci-bacilli, many being within leucocytes. The hamorrhagic area of the spleen contained a few bacilli almost entirely within leucocytes. The blood was sterile.

Rabbit ${ }^{1}{ }^{1}-$ Full-grown wild rabbit, inoculated in the thigh with $0.25 \mathrm{cc}$. three-days-old broth subculture from Guinea-pig 5.

Twenty-four hours after, beyond a considerable swelling at the point of inoculation, the animal was apparently healthy. Death evidently occurred about thirty-six hours after inoculation, the rabbit being found dead with rigor mortis well advanced at forty-two hours.

Post-mortem Examinatzon.-Animal in fine condition. Large quantity of subcutaneous œdema in inoculated thigh, the cedema extending for about $I$ in. along the floor of the abdomen. The viscera were normal, but the heart exhibited a rupture one-third inch long around the apex of the left ventricle, the pericardium containing a quantity of clotted blood.

Microscopical Examination.-A few organisms in the subcutaneous œdema, very few of which were within leucocytes; none present elsewhere. Pure cultures were obtainable from the cedema.

Rabbit 2.-Fed with $0.75 \mathrm{cc}$. three-days-old broth subculture from Guinea-pig 5 .

No very apparent symptoms were observed, the animal remaining crouched in the corner of the hutch, as is usual with wild rabbits when in confinement, and eating but sparingly. Found dead on the morning of the sixth day after inoculation.

Post-mortem Examination.-Animal in poor condition; stomach full and normal; intestines empty, but normal; liver congested, and exhibiting beneath the capsule a number of small circular abscesses the size of a pin-head, the deeper portion of the liver substance being normal ; the kidneys contained a number of similar nodules beneath the capsule. The spleen and the lymphatic glands of the abdomen were normal. Thorax: Acute fibrinous pleurisy, the pulmonary and costal pleura being adherent throughout. Here and there caseous foci of a very faint greenish tint and fairly solid consistency were present. Both lungs were congested throughout. The large posterior lobes of both lungs contained caseous areas surrounded by zones of pneumonia. The middle lobe of the left lung was dark and firm in consistency, evidently the second stage of pneumonia, but no caseating areas were present. The pericardium was inflamed with fibrinous effusion. The thoracic lymphatic glands were congested, but otherwise normal.

Microscopical Examination. - The characteristic organisms were found in large numbers in the caseous necrotic pulmonary areas and the pleuritic and pericardial effusions, while a small number were

1 All the rabbits used in these experiments were of the wild variety. 
present also in the small greyish caseous nodules in the liver and kidney. No organisms were present in the blood.

Rabbit 3.- Inoculated in the loin with $0.25 \mathrm{cc}$. three-days-old broth subculture from Rabbit I.

Forty-eight hours after inoculation animal well, with only slight swelling at inoculation point. Twelve hours later evidences of illness were exhibited, total loss of appetite, shivering and trembling, limp when raised, and quite tame. Death occurred at seventy-one hours after inoculation, quietly and without struggling.

Post-Mortem Examination.-At seat of inoculation a small area of caseous material surrounded by a zone of extravasated blood extended from the ilium to the middle of the thorax and downwards for about 2 in. Particularly at the flanks and axilla there was present subcutaneously a quantity of gelatinous cedema, with small areas of blood extravasation. The peritoneum contained a large amount of sero-sanguineous semi-gelatinous effusion. The large intestines were mottled with sub-peritoneal petechiæ, and the liver congested. Other organs normal.

Microscopical Examination.-Subcutaneous caseous material contained masses of characteristic bacilli, chiefly of a very short variety and frequently in cells. A few bacilli were present in the subcutaneous exudate. Media inoculated with blood and spleen pulp remained sterile.

Rabbit 4.-Inoculated in loin with $O^{\circ}$ I cc. three-days-old broth subculture from rabbit 3 .

This animal gradually developed, without exhibiting any evidences of general disturbance, a soft, movable, subcutaneous tumour at the seat of inoculation. Fourteen days after inoculation this tumour had attained to about the size of a walnut, and to the touch appeared round and circumscribed. Its presence did not appear to inconvenience the animal. On this date the animal was reinoculated in the thigh with $0.25 \mathrm{cc}$. broth subculture from guinea-pig 4 (see previous rabbits $I$ and 2 , which received earlier broth culture from same guineapig). Eight days after the second inoculation (twenty-two days after the first) this rabbit was found dead.

Post-mortem Examination.-Animal emaciated. At the seat of inoculation in the loin was present a circumscribed triangular abscess extending from the ilium forward to the third last rib, where it ended in a blunt apex. Along the inferior edge were numbers of small pea-shaped nodules. The walls of these abscesses were thin and friable. The inoculated limb showed a much more extensively diseased condition. Masses of pus of a greyish colour extended throughout the whole of the subcutaneous region of the limb, from the tarsal joint upwards to the base of the tail. These caseous areas were very irregular in outline, and varied in form, being from oneeighth inch to half-an-inch in diameter, and extended to the fibrous tissue between the muscles. Here, again, the surrounding membrane was thin and friable. The pelvic glands were caseated. Other organs normal.

Microscopical Examination demonstrated the presence of the characteristic organism in the caseous material.

Calf (zell grown, Six Months old).-Inoculated in thigh with $1 \mathrm{cc}$. liquid serum subculture from rabbit 3 . 
Next day the animal was evincing great pain in the inoculated limb, being barely able to move. There was considerable swelling, hot and painful, above the seat of inoculation. Recovery was rapid, however, and in three days, though a slight swelling still remained, the animal was practically normal. Ten weeks later the animal was killed for examination, when, but for a small nodule the size of a bean composed of fibrous tissue, near the seat of inoculation, there was no evidence of disease.

Rabbit 5.-Fed with $0.02 \mathrm{cc}$. broth subculture from rabbit 3 .

Killed two months later and found normal.

Rabbit 6.- Inoculated in loin with $O^{\circ} \mathrm{I} \mathrm{cc}$. three-days subculture in liquid serum from rabbit 3 .

This animal rapidly developed a large swelling at the seat of inoculation, until, by the twelfth day, it had reached the size of a small orange. It was then killed for examination.

Post-mortem Examination.-Animal in fair condition. Situated between the muscles of the loin was a large caseous abscess about the size of a mandarin orange. The wall of the abscess was thin and readily ruptured. The pus was very thick, almost caseous, with a very faint greenish tint. All organs normal.

Microscopical Examination.-The pus from near the wall of the abscess contained numbers of very short and irregular bacilli, while that from the centre of the mass was almost free of organisms. Scrapings from the periphery of the abscess contained more characteristic bacilli, they being larger and more distinct. Serum-tubes inoculated from the centre of the abscess remained sterile.

Rabbit 7.- Inoculated in loin with $O^{\circ}$ I cc. liquid serum subculture three-days-old from rabbit 3 at the same time as rabbit 6 .

A swelling at the seat of inoculation resulted, gradually extending subcutaneously downwards to nearly the medial abdominal line, forming a soft circumscribed round band about $I$ inch in breadth. By the fourteenth day this belt-like swelling was very distinct, especially when the animal was raised by the ears. The animal continued to eat well, and appeared otherwise in fair health. The tumour at the seat of inoculation increased gradually. On the morning of the nineteenth day the rabbit was found dead.

Post-mortem Examination.-Animal emaciated. The swelling as above was found to consist of a yellowish (faintly green) caseous material, with little or no defined wall. The pus extended from the region of the Join (inoculation-seat), where there was a mass the size of a small orange, downwards in a broad raised band I inch in diameter to almost the posterior extremity of the thorax. The caseous material and the wall of the abscess were similar to that of rabbit 6 . Other organs were normal.

Microscopical Examination resulted as in the previous experiment.

Rabbit 8.-Fed with $0.5 \mathrm{cc}$. same culture as used for Nos. 6 and 7 .

When killed two months later found quite normal.

Guinea-pig 6.-Inoculated with $0.5 \mathrm{cc}$. five-days-old broth subculture from rabbit 7 .

Gradually a swelling developed above the seat of inoculation; the animal became poorer in condition, but the appetite remained normal till before death, which occurred, with little or no premonition, on the twenty-ninth day after inoculation. 
Post-mortem Examination.-The thigh, above the seat of inoculation, contained an enormous abscess about $I_{2} \frac{1}{2}$ inch long by $I$ inch broad. The centre consisted of a mass of greyish purulent matter with a very faint greenish tint, surrounded by a very thin capsule. Liver: At the inferior border distributed amongst the different lobes were twelve circular abscesses varying from the size of a pea to a small marble. Two of these small nodules were firmly attached to the peritoneal surface of the posterior two ribs. In the body of the liver four larger abscesses were situated. These nodules consisted of a very thin wall adherent to the liver-tissue, and a purulent grey degenerated material in the centre. Thorax: Pleural cavity contained about $4 \mathrm{cc}$. clear serous effusion. Other organs normal.

Microscopical Examination demonstrated the presence of the specific organisms only in the pus and caseous material.

\section{THE DISEASE IN AUSTRALIA.}

A few years ago Drs Cherry and Bull, of Melbourne, published a pamphlet on caseous lymphatic glands (pseudo-tuberculosis) in sheep. (See also Veterinarian, Vol. 1xxii., p. 523.)

The disease in every way appears to be the same as that described here, while the bacilli isolated have the same characteristics.

\section{Comparison with Organism received from Australia.}

When in Melbourne, Mr Reakes, Assistant Chief Veterinarian, obtained from Drs Cherry and Bull culture-tubes inoculated at the time with the caseous material from abscesses. Culturally and morphologically the Australian and New Zealand organisms are similar. Experiments were made to ascertain the relative virulence.

Rabbit 9.--Inoculated in the loin with $\mathrm{O}_{4} \mathrm{cc}$. six-days-old broth subculture of the Melbourne bacillus.

Rabbit I0.-Fed with I cc. of the same culture.

Rabbit I I.-Inoculated in the loin on the same date with $0_{4} \mathrm{cc}$. five-days-old broth subculture from rabbit 7 .

Rabbit 12.-Fed with I cc. of the same culture.

Gradually in both inoculated rabbits ( 9 and II) the typical swelling at the seat of inoculation occurred, until, on the seventh day, the pus had burrowed downwards, forming a raised soft band from the loin to almost the median abdominal line, although the swelling in 9 never quite reached the dimensions of that of I I. On the seventeenth day the abscesses of both were found to have burst and the swellings to be decreasing. Later on a gradual recovery took place, which, however, was the more marked in 9 than in II. Fifty-three days after inoculation both animals were killed for examination.

Post-nortem Examination-Rabbit 9 was found to have completely recovered, there being no trace of any lesion in the body after the skin was removed. The skin itself showed at the seat of inoculation a small flat nodule quarter inch in diameter composed solely of fibrous tissue. Rabbit I I showed subcutaneously near the seat of inoculation five nodules varying from one-third inch to I inch in diameter, each consisting of a dense fibrous wall enclosing greyish caseous material. The post-scapular lymphatic gland was enlarged to the 
size of a marble, and presented a similar appearance on section to the subcutaneous nodules.

It will thus be noted that, although the organism with which I have experimented throughout in all my series had considerably decreased in virulence by the time I made this comparative experiment, it was yet more virulent than that received from Australia.

The feeding-experiments on rabbits 10 and $\mathrm{I} 2$ failed to induce any diseased condition. Both rabbits were killed six weeks after being fed, and were found practically normal. (Compare previous feedingexperiments on rabbits.)

\section{SUMMARY OF EXPERIMENTS.}

These experiments have been chronicled in the order in which they were conducted. Were each class of animal to be kept by itself, as is usual, the gradual decrease of virulence would not be so obvious. The disease affects sheep, goats, guinea-pigs, and rabbits, but cattle appear to be immune. It will be observed that the early cultures with which the experiments were made were very virulent to guinea-pigs, rabbits, and even sheep. Sheep 2 succumbed in 36 hours, guinea-pigs $I, 2,3$, and 5 in two to three days, and rabbits 1 and 3 in 36 and 71 hours respectively.

In the case of the early guinea-pigs and sheep 2 the gastric lesions are important, no such conditions having been observed in the more chronic cases, or in the rabbits. The evident diminution of virulence, particularly on passage through rabbits, is also notable.

The progress of the disease from lymph-gland to lymph-gland, and the ultimate infection of the lung if death be delayed, is somewhat similar to the course of true tuberculosis. With the exception of rabbit 2, in which typical pulmonary and pleural lesions were induced, efforts to produce the disease by the alimentary tract were unsuccessful. On the contrary, provided death does not rapidly ensue from probably the formation of a powerful toxin (as in sheep 2 and the early guinea-pigs and rabbits), and provided a sufficient time elapses, the lung ultimately becomes affected even when the furthest extremity of the body is inoculated.

The distinctly green colouration of the pus in sheep does not appear to be due to any chromogenic action on the part of the bacillus, but rather to the reaction on the part of the ovine system. (Compare the case of general pyæmia due to a streptococcus and the case of pulmonary actinomycosis in this report.)

The similarity in the pathogenic action of the bacillus to that of the tubercle bacillus is important in many respects, notably the general appearance of the nodules, the progression of the disease from gland to gland with the ultimate affection of the lung, and it is for that reason I prefer the term "pseudo-tuberculosis "(though admittedly open to many objections, not the least being that other diseases have been classed under the same term). In rabbits the pus and caseous material at the most only showed the faintest tinge of green colour; frequently it could not be detected.

The similarity of the organism culturally, particularly the yellow appearance on ox-serum, the sparse growth at first on ordinary agar, and the growth on broth to the diphtheria bacillus, is worthy of note. 


\section{OTHER INVESTIGATIONS.}

Norgaard and Mohler (Report of U.S. Bureau of Animal Industry, I 899) describe what is apparently the same disease under the heading of "Ovine Caseous Lymphadenitis." Morphologically the bacillus isolated appears to agree in most respects with that I have described. Judging by the experiments quoted, however, the bacillus seems to have been less virulent than the one experimented with here, notably in the earlier stages of the experiment. In rabbits subcutaneous inoculations did not result in death until the thirtieth to the fortyeighth day, and even intra-abdominal inoculations were less rapidly fatal than the subcutaneous inoculations here. Feeding-experiments were always negative in results. In sheep and in guinea-pigs the results of experiments appear to show that the organism even with larger doses was less virulent. In America the disease appears to affect sheep to much the same extent as it does here, although the pulmonary and pleural lesions do not appear to be so common, which may be due in a great measure to the two conditions not being associated in the minds of Inspectors of meat. The percentage of sheep condemned in the abattoirs, \&c., of the United States of America, seems to be practically similar to the percentage we have found here.

Nocard has described a disease, under the head of "Lymphangitis simulating Farcy," in horses, due to a bacillus the morphological and cultural characteristics of which are very similar to the organism already described. It is particularly on the gelatinised serum, however, that the similarity is so striking as to leave no doubt that the two microbes are identical. Nocard found this disease clinically similar to farcy (form of glanders) to be due to the presence of this microbe in sixteen cases out of sixty-seven horses suspected of being affected with glanders. ${ }^{1}$

In I89r what appears to have been the same disease of sheep in Europe was studied by Priesz, and although I have no opportunity of consulting his memoirs on the subject, it would appear from Norgaard and Mohler that he described completely the above bacillus.

\section{THE PATHOGENESIS AND PROPHYLAXIS OF THE ACCIDENTS FOLLOWING PROTECTIVE INOCULA- $\operatorname{TION}_{x}{ }^{2}$}

\section{By M. E. Leclainche, Alfort, and M. H. Vallee, Toulouse.}

ALL the methods of immunisation by means of attenuated virus are liable to accidents, and although the general percentage of these is not at all great they cannot be altogether neglected. Moreover, the accidents are very unequally distributed, and when they occur they may be the cause of considerable loss to individual owners.

The same facts are observed in the case of the three diseases against which it is possible to vaccinate by using a modified virus, viz., anthrax, swine-erysipelas, and quarter-evil.

\footnotetext{
1 "Annales de l'Institut Pasteur," Tome X., p. 609.

2 Translated from the "Annales de l'Institut Pasteur," August 1902.
} 